Figure 1

Spectrum of VR technologies used in this study. From left to right: Oculus Quest, Oculus Go, Gear VR, Oculus Rift S.

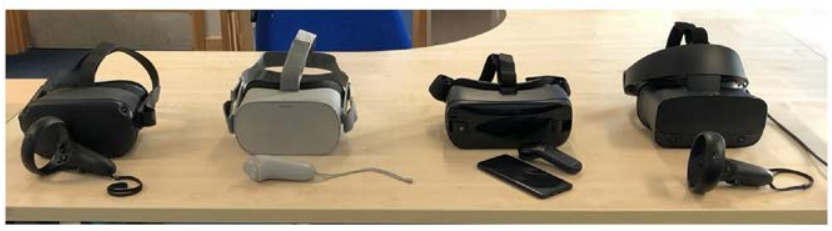

Acknowledgements: I would like to acknowledge the contributions of the staff working within the Rheumatology department at the Norfolk and Norwich University Hospital. I would also like to thank and acknowledge our participants for being involved in the study.

Disclosure of Interests: Jordan Tsigarides Grant/research support from: Our digital health industry partners (Orbital Global) provided a small financial contribution to support this study., Vanessa Grove: None declared, Dheeraj Sethi: None declared, Jacqueline Chipping: None declared, Susan Miles: None declared, Nicholas Shenker: None declared, Saber Sami: None declared, Alex MacGregor: None declared.

DOI: 10.1136/annrheumdis-2021-eular.2017

\section{POS0019 PREDICTORS OF SUSTAINED 6 MONTHS REMISSION AND FLARE IN PATIENTS WITH POLYMYALGIA RHEUMATICA}

C. Perricone $^{1}$, E. Fiumicelli ${ }^{1}$, G. Cafaro ${ }^{1}$, R. Bursi ${ }^{1}$, R. Ilenia ${ }^{1}$, E. Bartoloni Bocci ${ }^{1}$, R. Gerli ${ }^{1}{ }^{1}$ Rheumatology, Department of Medicine and Surgery, Perugia, Italy

Background: Polymyalgia rheumatica (PMR) is a typical inflammatory disease of the elderly. The mainstay of treatment is usage of oral glucocorticoids (GC) usually leading to a dramatic response. However, long-term treatment is often required. In some instances, remission cannot be achieved and disease flares can occur. To date, few papers investigated the predictive factors of response to GC therapy suggesting a role for weight, elevated plasma viscosity and C-reactive protein (CRP). Moreover, there are no data on predictor factors of disease flares after 6 months of sustained remission.

Objectives: To evaluate the factors predictive of sustained 6 months remission and of flare in patients with PMR.

Methods: We evaluated clinical data from 137 PMR patients treated with GC for more than 6 months between September 2002 and June 2020. Patients were evaluated at baseline, re-evaluated at 1 month and after 3 months and then at least every 3 months. We analyzed the differences between patients who achieved remission within 6 months of diagnosis, who maintained it for at least 6 months, and patients who did not. Remission was defined as 1) the absence of clinical symptoms, 2) $<7.5 \mathrm{mg} /$ day of prednisone equivalents and 3) negativity of ESR and CRP. Patients treated with methylprednisolone were excluded from the analysis.

Results: Among the 137 patients (baseline F68: M69, mean age \pm SD $74.5 \pm 6.6$ years, mean ESR \pm SD $49.9 \pm 27.7 \mathrm{~mm} / \mathrm{h}$, mean $C R P \pm S D 6.1 \pm 11.4 \mathrm{mg} / \mathrm{dl}), 57$ achieved remission at 6 months (41.6\%), and after this period, another 47 went into remission for a total of 104 patients $(75.9 \%)$. The months required to achieve remission averaged $6.4 \pm 3$. No differences were observed between patients who achieved remission and patients who did not regarding age, sex, CRP, starting dose of GC, anti-CCP and rheumatoid factor. The ESR at baseline was higher in patients who did not achieve remission $(58.1 \pm 33.4 \mathrm{~mm} / \mathrm{h})$ than in patients who achieved it $(46 \pm 24.8 \mathrm{~mm} / \mathrm{h}, \mathrm{p}=0.06)$. ESR at baseline correlated with the time needed to reach normalization of both ESR and CRP $(p=0.01$, Figure 1). Thirty-six of 104 patients (34.6\%) had a disease flare after a mean of $6.1 \pm 2.7$ months of remission. The mean GC intake at flare was $2.8 \pm 3 \mathrm{mg} /$ day. Only $15 / 36(41.6 \%)$ patients were able to withhold GCs after flare, compared with $48 / 68$ (70.5\%) patients who achieved remission and did not experience flare. There were no significant differences in any analyzed parameters between patients who experienced flares and those who did not, including ESR and CRP values at baseline $(50.3 \pm 26.7 \mathrm{~mm} / \mathrm{h}$ and $6.8 \pm 12.1 \mathrm{mg} / \mathrm{dl}$, respectively). A higher percentage of patients who did not achieve remission experienced flare than patients who went into remission $(p=0.009)$.

Conclusion: Achieving complete remission at 6 months occurs in a consistent percentage of patients with PMR. Despite this, a significant percentage of patients may need prolonged steroid treatment and may experience flare, even after 12 months of follow-up. Remission at 6 months is likely to be a good predictor of remission at 12 months, as already reported in recent studies $(1,2)$. Elevated ESR at diagnosis appears to be a negative predictor for remission at 6 months. Patients who achieve complete remission are less likely to develop flare. True remission should coincide with the discontinuation of steroid treatment; in fact, the maintenance of steroid therapy, even at low doses, is predictive of the development of flare.

\section{REFERENCES:}

[1] Hattori K, et al. Int J Rheum Dis. 2020

[2] Camellino D, et al. Nat Rev Rheumatol. 2020

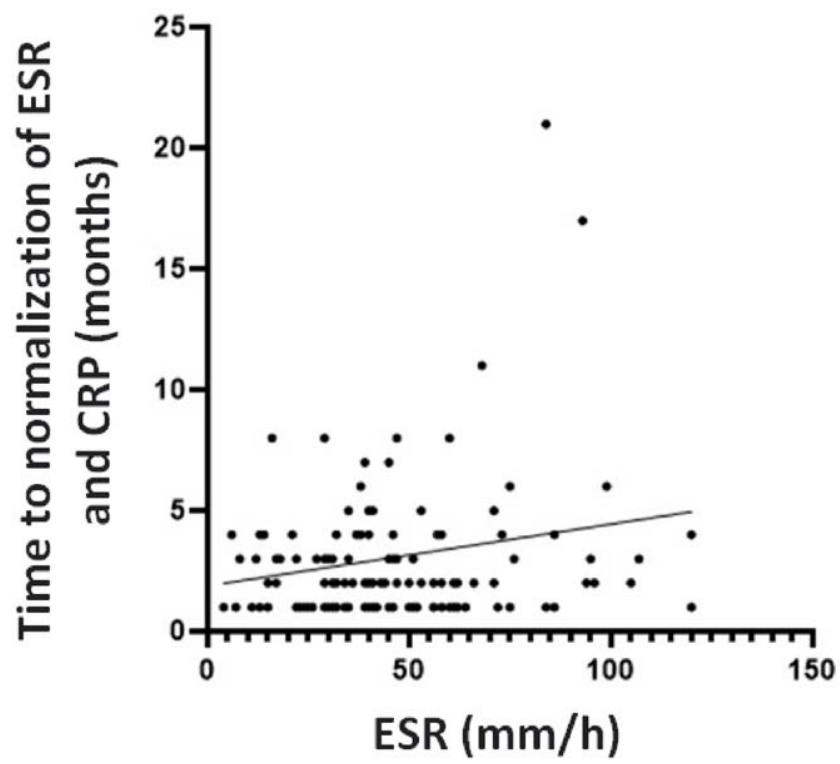

Figure 1.

Disclosure of Interests: Carlo Perricone Speakers bureau: BMS, Lilly, Celgene, UCB, Abbvie, Pfizer, Sanofi, Accord, Novartis, Elena Fiumicelli: None declared Giacomo Cafaro: None declared, Roberto Bursi: None declared, Ricucci Ilenia: None declared, Elena Bartoloni Bocci: None declared, Roberto Gerli: None declared.

DOI: 10.1136/annrheumdis-2021-eular.3593

\begin{tabular}{|l|l}
\hline POSO020 & EFFICACY AND SAFETY OF SECUKINUMAB IN \\
PATIENTS WITH ROTATOR CUFF TENDINOPATHY: A \\
24-WEEK, RANDOMISED, DOUBLE-BLIND, PLACEBO- \\
CONTROLLED, PHASE II PROOF-OF-CONCEPT TRIAL
\end{tabular}

N. L. Millar ${ }^{1}$, I. Mcinnes ${ }^{1}$, L. Mindeholm ${ }^{2}$, A. Seroutou ${ }^{3}$, J. Praestgaard ${ }^{4}$, U. Schramm ${ }^{2}$, R. Levitch ${ }^{5}$, E. Weber ${ }^{6}$, D. Laurent ${ }^{7}$, J. Rosen ${ }^{8}$, G. Schett ${ }^{9}$, R. Roubenoff ${ }^{10}$, M. Schieker ${ }^{2} .{ }^{1}$ Institute of Infection, Immunity \& Inflammation, University of Glasgow, Glasgow, United Kingdom; ${ }^{2}$ Novartis Institutes for BioMedical Research, TM MSD Bone, Joint and Tendon, Basel, Switzerland; ${ }^{3}$ Novartis Institutes for BioMedical Research, Biostatistics, Basel, Switzerland; ${ }^{4}$ Novartis Institutes for BioMedical Research, Data Science, Cambridge, United States of America; ${ }^{5}$ Novartis Institutes for BioMedical Research, Cardio-renalmetabolic, Basel, Switzerland; ${ }^{6}$ Novartis Institutes for BioMedical Research, MSD LAB WEBER, Basel, Switzerland; ${ }^{7}$ Novartis Institutes for BioMedical Research, BMD Clincal \&Transplant Imaging, Basel, Switzerland; ${ }^{8}$ New York Presbyterian Queens, Department of Orthopaedics \& Rehabilitation, New York, United States of America; ${ }^{9}$ University of Erlangen-Nuremberg, Department of Internal Medicine 3, Erlangen, Germany; ${ }^{10}$ Novartis Institutes for BioMedical Research, Translational Medicine, Basel, Switzerland

Background: Rotator cuff tendinopathy (RC TP) is a multifactorial condition and one of the most common causes of musculoskeletal burden. Current standard of care $(\mathrm{SoC})$ is limited to pain relief with NSAIDs and physiotherapy. Recent evidence indicates that IL-17A-expressing tendon-resident immune cells are present in human overuse tendinopathy, and IL-17A levels are increased in early human tendinopathic tissue samples $[1,2]$. Secukinumab (SEC) is a fully human, monoclonal antibody that binds to and neutralises IL-17A.

Objectives: To evaluate the efficacy and safety of SEC in patients with active overuse RC TP refractory to oral NSAIDs/acetaminophen, physiotherapy or corticosteroid injections.

Methods: 96 patients with symptomatic RC TP with no or $<50 \%$ rupture were randomly assigned to receive seven subcutaneous injections of SEC $300 \mathrm{mg}$ o placebo (PBO) at baseline and Weeks 1, 2 and 3, followed by every 4 weeks starting at Week 4 . The primary endpoint was change from baseline in the Western Ontario Rotator Cuff (WORC) index score at Week 14 for SEC vs PBO (twosided $p<0.1$ ). Secondary endpoints included, visual analogue scale (VAS) pain score, Disability of Arm, Shoulder and Hand Questionnaire (QuickDASH) score, 